\title{
Association of Endothelial Nitric Oxide Synthase (eNOS) G894T Polymorphism With High Altitude Pulmonary Edema Susceptibility: A Meta-Analysis
}

\author{
Yongjun Luo, PhD; Yu Chen, MD; Yao Zhang, PhD; Qiquan Zhou, MD; Yuqi Gao, MD \\ From the Departments of High Altitude Diseases (Drs Luo and Zhou) and High Altitude Pathophysiology (Drs Chen and Gao), College of \\ High Altitude Military Medicine, Third Military Medical University; the Key Laboratory of High Altitude Medicine (Third Military Medical \\ University), Ministry of Education (Drs Luo, Chen, Zhou, and Gao); the Key Laboratory of High Altitude Physiology and High Altitude \\ Diseases (People's Liberation Army) (Drs Luo, Chen, Zhou, and Gao); and the Department of Epidemiology, Faculty of Preventive Medicine, \\ Third Military Medical University (Dr Zhang), Chongqing, China.
}

\begin{abstract}
Background.-High altitude pulmonary edema (HAPE) is a potentially deadly disease associated with exposure to altitudes greater than $3000 \mathrm{~m}$. Individuals who have previously experienced HAPE are at a significantly higher risk of recurrence, suggesting an underlying genetic component to HAPE pathogenesis. In a previous nuclear genomic study of individual variation in susceptibility to HAPE, the endothelial nitric oxide synthase $(e N O S)$ gene G894T polymorphism was identified as being associated with HAPE. However, another study found no association. Because of the low incidence of HAPE, sample sizes in current reports have been relatively limited. In this study, the association between the eNOS G894T polymorphism and HAPE was assessed through a meta-analysis of published data.

Methods.-The literature was searched in PubMed, Web of Science, and Embase for papers published before July 15, 2011. A fixed-effects model and a random-effects model were applied (Revman 5.0) on the basis of heterogeneity, and study quality was assessed in duplicate.

Results.-Five studies with 360 HAPE patients and 469 control subjects were analyzed. There were no significant differences between carriers of the eNOS 894G and 894T polymorphism alleles in terms of the risk of developing HAPE.

Conclusions.-The eNOS $894 \mathrm{G}$ and $894 \mathrm{~T}$ polymorphism alleles are not associated with HAPE incidence.
\end{abstract}

Key words: HAPE, eNOS, G894T polymorphism, meta-analysis

\section{Introduction}

High altitude pulmonary edema (HAPE) is a potentially fatal disease that develops shortly after exposure to altitudes greater than $3000 \mathrm{~m}$. It is exacerbated by hypoxic, cold environmental conditions and fatigue. ${ }^{1}$ A principal characteristic of HAPE is exaggerated hypoxic pulmonary vasoconstriction. Oxygen sensors in the pulmonary vasculature detect changes in alveolar oxygen tension and modulate the caliber of small pulmonary arteries. In hypoxia, vasoconstriction is stimulated to redistribute

Disclaimer: The authors declare no conflicts of interest, financial or otherwise, pertaining to the publication of this article.

Corresponding author: Prof Yuqi Gao, College of High Altitude Military Medicine, Third Military Medical University, Chongqing 400038, PR China (e-mail: gaoy66@yahoo.com). blood flow to more effective areas of gaseous exchange. ${ }^{2}$ Many genes are known to contribute to pulmonary vascular tone. For example, endothelial nitric oxide synthase (eNOS) generates nitric oxide (NO). eNOS gene expression is upregulated in conditions that require reducing the alveolar to arterial oxygen tension difference. ${ }^{3}$ Many studies have focused on single-nucleotide polymorphisms (SNPs) of eNOS, to find an association between polymorphisms and HAPE. ${ }^{3-7}$ However, because HAPE is rare, only limited data are currently available. G894T $(e N O S 894 \mathrm{G} \rightarrow \mathrm{T})$ is the most common polymorphism of $e N O S,{ }^{3-7}$ and correlates with plasma NO levels. ${ }^{8}$ Some studies have reported that the eNOS G894T polymorphism is related to HAPE susceptibility, whereas others report no association. ${ }^{3-5}$ To systematically address this issue, we performed a meta-analysis of published studies 
to assess the association of the eNOS G894T polymorphism with HAPE.

\section{Methods}

\section{LITERATURE SEARCH}

We searched all papers published before July 15, 2011, in PubMed, Web of Science, and Embase databases. The key words were "polymorphism," "High Altitude Pulmonary Edema," "SNP" and "HAPE," and "HAPE." Articles not written in English were excluded, as were abstracts and unpublished reports.

\section{INCLUSION AND EXCLUSION CRITERIA}

Inclusion criteria were as follows: 1) case-control studies to evaluate the association between the eNOS G894T polymorphism and HAPE risk; and 2) useful data containing the genotype number or frequency. ${ }^{9}$ Exclusion criteria were studies with insufficient genotype number or frequency.

\section{DATA EXTRACTION}

For each study, information extracted included first author, year of publication, study population, country, and genotype number of cases and controls. If multiple data were available for different times or altitudes in the same population, we selected the highest altitude or longest study time, using data containing the genotype number or frequency.

\section{STATISTICAL ANALYSIS}

In this meta-analysis, we assessed association of the eNOS $894 \mathrm{G}$ allele and HAPE compared with the T allele (allelic model), and used the dominant genetic model to compare genotypes GG vs GT + TT, GG + GT vs TT, GG vs TT, GT vs GG, and TT vs GT. The alleles and genotypes between patients and control subjects were compared by the unadjusted odds ratios (OR) and $95 \%$ confidence interval (CI). The unadjusted OR is not the same as relative risk, but is a measure of how much of the difference between 2 groups is explained by a given variable. The heterogeneity assumption was assessed by a $\chi^{2}$-based $\mathrm{Q}$ test and $\mathrm{I}^{2}$ test. The heterogeneity was considered significant when the probability value was less than .05 . If there was no heterogeneity $(P>.05)$ among the studies, the pooled OR estimate of each study was calculated by a fixed-effects model $(P<.05){ }^{10,11}$ If heterogeneity was present, we used a random-effects model to account for interstudy heterogeneity instead of using the fixed-effect model (Mantel-Haenszel). ${ }^{12}$ All statistical analysis was performed using Revman 5.0 (Cochrane IMS, Copenhagen, Denmark).

Publication bias was investigated using a funnel plot in which the standard error of $\log (\mathrm{OR})$ of each study was plotted against its OR. An asymmetric plot suggested possible publication bias. Funnel plot asymmetry was assessed using Egger's linear regression test, which uses a linear regression approach to measure funnel plot asymmetry on the natural logarithm scale of the OR. ${ }^{13}$ The significance of the intercept was determined by a Student's $t$ test, as suggested by Egger. A probability value of less than .05 was considered representative of significant publication bias.

\section{Results}

\section{DESCRIPTION OF STUDIES}

Five studies with 360 HAPE patients and 469 control subjects were subjected to a meta-analysis. Baseline characteristics for the studies are in Table 1. Four studies were conducted in Asians, and one in Europeans. Sample sizes ranged from 91 to $309 .^{3-7}$

\section{META-ANALYSIS RESULTS}

We found no heterogeneity when comparing the genotypes G vs T, GG vs GT + TT, or GT vs GG (Table 2). A random effects model was used for analysis. Supplementary Figure 1 shows the pooled analysis of G894T

Table 1. Baseline characteristics of studies in the meta-analysis

\begin{tabular}{|c|c|c|c|c|c|c|c|c|c|c|c|}
\hline \multirow[b]{2}{*}{ Author } & \multirow[b]{2}{*}{ Year } & \multirow[b]{2}{*}{ Country } & \multirow[b]{2}{*}{ Location/altitudes $(\mathrm{m})$} & \multicolumn{2}{|c|}{ Sample size } & \multicolumn{3}{|c|}{ Case } & \multicolumn{3}{|c|}{ Controls } \\
\hline & & & & Cases & Controls & $G G$ & $G T$ & $T T$ & $G G$ & $G T$ & $T 7$ \\
\hline Ahsan $^{4}$ & 2006 & India & 3500 & 60 & 72 & 36 & 22 & 2 & 25 & 45 & 2 \\
\hline Ahsan $^{3}$ & 2004 & India & 3400 & 59 & 136 & 22 & 35 & 2 & 105 & 29 & 2 \\
\hline Weiss $^{5}$ & 2003 & Germany & 4559 & 51 & 50 & 20 & 24 & 7 & 24 & 22 & 4 \\
\hline Droma $^{6}$ & 2002 & Japan & $2758-3190$ & 41 & 51 & 20 & 21 & 0 & 43 & 6 & 2 \\
\hline Sun $^{7}$ & 2010 & China & $4500-5072$ & 149 & 160 & 130 & 18 & 1 & 155 & 4 & 1 \\
\hline
\end{tabular}


Table 2. Summary odds ratios and $95 \%$ confidence intervals of $e N O S$ polymorphisms and HAPE

\begin{tabular}{|c|c|c|c|c|c|c|c|}
\hline No & Polymorphisms & Heterogeneity $P$ value & Model & OR & $95 \% C I$ & $P$ & Supplementary figure \\
\hline 1 & G vs $\mathrm{T}$ & $<.01$ & Random effects model & 0.52 & $0.23-1.14$ & .10 & 1 \\
\hline 2 & GG vs $\mathrm{GT}+\mathrm{TT}$ & $<.01$ & Random effects model & 0.43 & $0.14-1.35$ & .15 & 2 \\
\hline 3 & GT vs GG & $<.01$ & Random effects model & 2.46 & $0.71-8.51$ & .15 & 3 \\
\hline 4 & GG vs TT & .69 & Fixed effects model & 0.61 & $0.26-0.46$ & .27 & 4 \\
\hline 5 & $\mathrm{GG}+\mathrm{GT}$ vs TT & .78 & Fixed effects model & 1.34 & $0.58-3.07$ & .68 & 5 \\
\hline 6 & TT vs GT & .29 & Fixed effects model & 0.88 & $0.38-2.03$ & .76 & 6 \\
\hline
\end{tabular}

CI, confidence interval; OR, odds ratio.

allele association with HAPE. The OR summary under a random-effects model indicated significant differences in the risk of developing HAPE between carriers of the 894 $\mathrm{G}$ and $\mathrm{T}$ alleles (OR, 0.52; 95\% CI, 0.23 to $1.14 ; P=$ .10). Similarly, some association was seen for inheritance of genotypes (GG vs GT+TT; OR, 0.43; 95\% CI, 0.14 to $1.35 ; P=.15$; Supplementary Figure 2). Genotype GT vs GG showed some significance (OR, 2.46; 95\% CI, 0.71 to $8.51 ; P=.15$ ) between HAPE and the control group (Supplementary Figure 3).

Because heterogeneity was not found in a comparison of the genotypes GG vs TT, GG + GT vs TT, or TT vs GT, the pooled OR estimate of each study was calculated by the fixed-effects model. Genotypes showing no significant association were GG vs TT (OR, 0.61; 95\% CI, 0.26 to $1.46 ; P=.69$; Supplementary Figure 4 ), and $\mathrm{GG}+\mathrm{GT}$ vs TT $(\mathrm{OR}, 1.34 ; 95 \% \mathrm{CI}, 0.58$ to $3.07 ; P=$ .78; Supplementary Figure 5), genotype (TT vs GT) showed no statistical significance (OR, 0.88; 95\% CI,
0.38 to $2.03 ; P=.29)$ between HAPE and the control group (Supplementary Figure 6).

\section{PUBLICATION BIAS DIAGNOSTICS}

Funnel plots were performed to determine whether the literature showed a publication bias based on dominant genetic model data. The shape of the funnel plots revealed no evidence of obvious asymmetry for all comparisons (Figure). In this study, the results showed no any obvious evidence of publication bias $(P>.05)$.

\section{Discussion}

To our knowledge, this is the first meta-analysis of studies on the association of the eNOS G894T polymorphism with HAPE risk. Five studies of 360 patients and 469 control subjects were analyzed. Our results revealed no significant differences in risk of developing HAPE
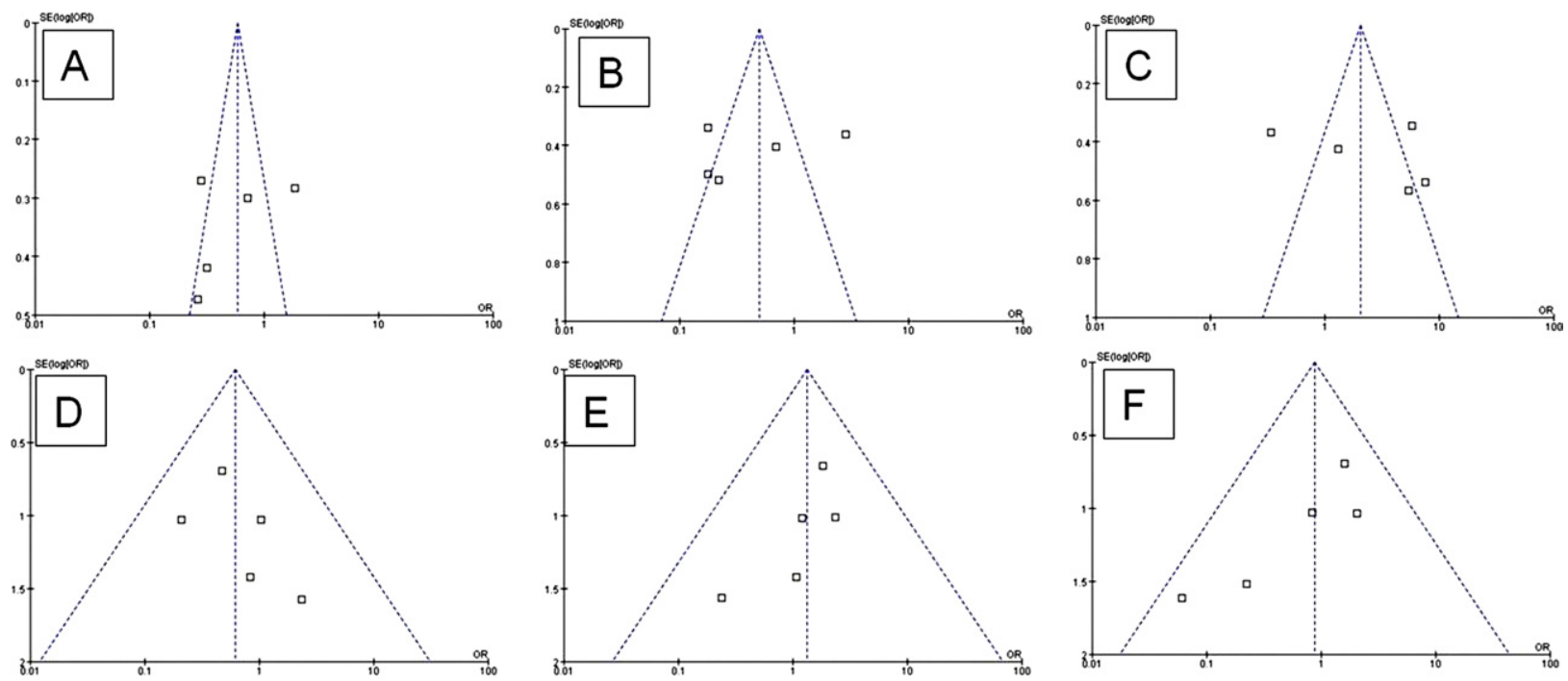

Figure. Funnel plot analysis for publication bias. Each point represents a separate study for the indicated association. A. Allele comparison (G vs T). B. $e N O S$ GG vs GT + TT. C. eNOS GT vs GG. D. eNOS GG vs TT. E. eNOS GG + GT. F. eNOS TT vs GT. (OR, odds ratio; SE, standard error.) 
between carriers of the eNOS 894G and 894T polymorphism alleles.

Individuals experiencing HAPE present with significantly lower levels of $\mathrm{NO}^{3}$ Impaired NO function might underlie the decreased physiologic tolerance to conditions experienced at high altitude. NO is a potent pulmonary vasodilator and regulates pulmonary vascular tone in humans. ${ }^{14}$ Release of NO in response to increased flow across the endothelium has been demonstrated in vitro. ${ }^{15} \mathrm{NO}$ inhalation therapy has been used successfully to treat HAPE. ${ }^{1}$ Inhaled NO causes a selective increase in perfusion, which improves ventilation-perfusion imbalance. ${ }^{16}$ The $894 \mathrm{G} / \mathrm{T}$ variant is located in exon 7 of the eNOS gene, and no significant difference in NO level is associated with G894 or T894 variants. ${ }^{7}$ Therefore, the involvement of the $894 \mathrm{G} / \mathrm{T}$ polymorphism in eNOS regulatory activity is a possibility. Also likely is a polymorphism in another gene that shows linkage disequilibrium with the $894 \mathrm{G} / \mathrm{T}$ variant. Although some reports indicate that the eNOS genotype and alleles do not affect circulating NO levels among healthy males, 894G/T might affect the response to hypoxia rather than baseline NO levels in healthy males. The 894G/T polymorphism was thought to be only a genetic marker associated with an unknown locus. ${ }^{17}$ Nonetheless, the positive association of two eNOS haplotypes with HAPE susceptibility suggests a possible interaction of multiple genetic markers in a haplotype that may be a major determinant of HAPE susceptibility. ${ }^{8,17,18}$ In this study, there was no association between $e N O S$ haplotypes and HAPE susceptibility. We did not find that the homozygous TT genotype was associated with higher HAPE risk, because of the low frequency of TT in the references. Further studies are necessary to confirm this hypothesis.

There are several limitations to this study. First, 4 of the 5 studies had Asian subjects and 1 had European subjects, and the TT genotype was rare. Second, the relatively small number of eligible studies prevented us from performing subgroup analyses to explore the effects of other confounders such as gender and age. In addition, the studies were conducted at different altitudes (although this might be controlled for). Third, some studies used HAPE-susceptible (HAPE-S) subjects and some investigated actual HAPE patients. Only the study by Yujing et $\mathrm{al}^{7}$ used a cohort of patients at altitude and studied those with and without HAPE. Droma et $\mathrm{al}^{6}$ and Weiss et $\mathrm{al}^{5}$ compared HAPE-S subjects with nonHAPE-S subjects. Ahsan et $\mathrm{al}^{3}$ studied HAPE patients compared with lowlanders who had been at $3400 \mathrm{~m}$ or higher (presumably without HAPE) and highlanders living at $3400 \mathrm{~m}$. Ahsan et $\mathrm{al}^{4}$ studied HAPE-resistant lowlanders at sea level compared with HAPE patients at altitude. These might or might not have been HAPE-S patients.
We analyzed the association of the 894G/T polymorphism with HAPE, and found no significant differences in risk of developing HAPE between carriers of $e N O S$ $894 \mathrm{G}$ and $894 \mathrm{~T}$ polymorphism alleles.

\section{Acknowledgments}

This research was supported by grants from the 973 Project of China (No. 2006CB504101) and the National Natural Science Foundation of China (No. 30900715).

\section{Supplementary Data}

Supplementary Figures 1-6 associated with this article can be found in the online version at http://dx.doi.org/ 10.1016/j.wem.2012.03.007.

\section{References}

1. Hackett PH, Roach RC. High-altitude illness. $N$ Engl J Med. 2001;345:107-114.

2. Gao Y, Raj JU. Role of veins in regulation of pulmonary circulation. Am J Physiol Lung Cell Mol Physiol. 2005; 288:L213-L226.

3. Ahsan A, Charu R, Pasha MAQ, Norboo T, Afrin F, Baig MA. eNOS allelic variants at the same locus associate with HAPE and adaptation. Thorax. 2004;59:1000-1002.

4. Ahsan A, Mohd G, Norboo T, Baig MA, Pasha MAQ. Heterozygotes of NOS3 polymorphisms contribute to reduced nitrogen oxides in high-altitude pulmonary edema. Chest. 2006;130:1511-1519.

5. Weiss J, Haefeli WE, Gasse C, et al. Lack of evidence for association of high altitude pulmonary edema and polymorphisms of the NO pathway. High Alt Med Biol. 2003; 4:355-366.

6. Droma Y, Hanaoka M, Ota M, et al. Positive association of the endothelial nitric oxide synthase gene polymorphisms with high-altitude pulmonary edema. Circulation. 2002; 106:826-830.

7. Yujing S, Fang M, Niu W, et al. Endothelial nitric oxide synthase gene polymorphisms associated with susceptibility to high altitude pulmonary edema in Chinese railway construction workers at Qinghai-Tibet over 4500 meters above sea level. Chin Med Sci J. 2010;25:215-222.

8. Crawford DC, Nickerson DA. Definition and clinical importance of haplotypes. Annu Rev Med. 2005;56:303-320.

9. Dai L, Liu D, Guo H, Wang Y, Bai Y. Association between polymorphism in the promoter region of Interleukin 6 $(-174 \mathrm{G} / \mathrm{C})$ and risk of Alzheimer's disease: a metaanalysis. J Neurol. 2011;259:414-419.

10. Mantel N, Haenszel W. Statistical aspects of the analysis of data from retrospective studies of disease. J Natl Cancer Inst. 1959;22:719-748.

11. Ma X, Chen C, Xiong H, Li Y. Transforming growth factor $\beta 1 \mathrm{~L} 10 \mathrm{P}$ variant plays an active role on the breast 
cancer susceptibility in Caucasian: evidence from 10,392 cases and 11,697 controls. Breast Cancer Res Treat. 2010;124:453-457.

12. Petitti DB. Meta-Analysis, Decision Analysis, and CostEffectiveness Analysis: Methods for Quantitative Synthesis in Medicine. New York, NY: Oxford University Press; 1994.

13. Egger M, Davey Smith G, Schneider M, Minder C. Bias in meta-analysis detected by a simple, graphical test. BMJ. 1997;315:629-634.

14. Pepke-Zaba J, Higenbottam TW, Dinh-Xuan AT, Stone D, Wallwork J. Inhaled nitric oxide as a cause of selective pulmonary vasodilatation in pulmonary hypertension. Lancet. 1991;338:1173-1174.
15. Palmer RM, Ferrige AG, Moncada S. Nitric oxide release accounts for the biological activity of endothelium-derived relaxing factor. Nature. 1987;327:524-526.

16. Barberà JA, Roger N, Roca J, Rovira I, Higenbottam TW, Rodriguez-Roisin R. Worsening of pulmonary gas exchange with nitric oxide inhalation in chronic obstructive pulmonary disease. Lancet. 1996;347:436-440.

17. Nagassaki S, Metzger IF, Souza-Costa DC, Marroni AS, Uzuelli JA, Tanus-Santos JE. eNOS genotype is without effect on circulating nitrite/nitrate level in healthy male population. Thromb Res. 2005;115:375-379.

18. Johnson GC, Esposito L, Barratt BJ, et al. Haplotype tagging for the identification of common disease genes. Nat Genet. 2001;29:233-237. 